\title{
Influence of Native Video Advertisement Duration and Key Elements on Advertising Effectiveness in Mobile Feeds
}

\author{
Bingcheng Wang $\mathbb{D}^{D}$, Man Wu, Pei-Luen Patrick Rau $\mathbb{D}^{D}$, and Qin Gao \\ Department of Industrial Engineering, Tsinghua University, Beijing 100084, China \\ Correspondence should be addressed to Pei-Luen Patrick Rau; rpl@mail.tsinghua.edu.cn
}

Received 8 July 2020; Revised 10 November 2020; Accepted 15 November 2020; Published 2 December 2020

Academic Editor: Salvatore Carta

Copyright (C) 2020 Bingcheng Wang et al. This is an open access article distributed under the Creative Commons Attribution License, which permits unrestricted use, distribution, and reproduction in any medium, provided the original work is properly cited.

\begin{abstract}
This research investigated the influence of advertisement (ad) duration and key elements (titles, logos, and texts) on advertising effectiveness in mobile feeds. We recruited 40 participants (27 men and 13 women) who are aged from 20 to 43 years $(M=29.33$, $\mathrm{SD}=6.67$ ). The participants were assigned randomly to four groups to watch four different types of ads: 6-second ads with key elements, 15-second ads with key elements, 15-second ads without key elements, and 30-second ads without key elements. We measured advertising effectiveness from four aspects: users' attention, emotion, memory, and attitudes. During the experiment, a researcher recorded participants' electroencephalography and eye movements. After the experiment, participants were required to complete a questionnaire and were interviewed. Results showed that participants felt more positive when watching 6-second duration ads in mobile feeds than the 15-second and 30-second ads; however, their memory of the ads was worse. The participants paid more attention to the key elements rather than the content of the ads. This research elucidated the features of native video ads in mobile feeds and provided some useful suggestions for advertisers who design video ads.
\end{abstract}

\section{Introduction}

Mobile feeds involve customized aggregation of web content from multiple sources on mobile devices. Applications (apps) such as YouTube, Twitter, and Facebook generate feeds according to users' personal preferences and accurately push the content to the users. The contents, varying from a movie trailer to the latest news, are often ordered or grouped by time and subject and are presented dynamically in a stream or a list. Users can read the feed at any time to keep informed of their interests. Because of their customizability and flexibility, mobile feeds are becoming increasingly popular in our daily life, and many companies choose to advertise in users' mobile feeds.

Native advertising is one of the most popular and effective advertising methods in mobile feeds [1]. Native ads are designed to minimize disruption to the user's online experience, either by increasing its relevance for viewers or mimicking the surrounding content [2]. Sponsored stories on Facebook or promoted tweets on Twitter are two good examples of native advertising. Native ads usually disguise themselves in a form that matches the format or function of the user experience on the platform on which they are displayed $[3,4]$. The visual disguise of native ads can reduce the users' perceived intrusiveness and advertising avoidance and increase their willingness to read or watch the ads $[5,6]$. Further, with the help of big data and recommendation algorithms, advertisers will accurately deliver ads of high content relevance to their target users [7-9], thus increasing users' engagement and decreasing the postclick annoyance effect $[3,10,11]$. Given these advantages, mobile-feed ads are gaining increasing attention from businesses and in academia.

Native video ads in mobile feeds are becoming more common recently due to the popularity of short videos. Most studies have been focused on native ads presented in the form of text $[3,4,12,13]$, but native ads in the form of video have rarely been studied thoroughly. Compared to the native text ads (such as sponsored stories or promoted tweets), native video ads enjoy much more media richness $[14,15]$ 
but face several shortcomings when applying to mobile feeds. Firstly, the display area of the mobile video ads is usually minimal due to the relatively small size of mobile touchscreens. Most users tend to hold the devices in a portrait orientation when viewing feeds. Secondly, the duration of the video ads is usually too long for mobile users, and most are too impatient to watch the entire ad. However, simply shortening the video duration brings advertising effectiveness since it would lead to underexposure of the core elements of the ad [16]. Thirdly, video ads in mobile feeds are usually played silently, which results in poorer media richness comparing to traditional video ads [14, 17]. Thus, it is necessary to improve mobile-feed video ads to compensate for their shortcomings. One practical approach is to add some key elements to enhance users' perception of the ads, such as enlarging the texts or emphasizing keywords or logos. However, the effectiveness of these approaches has rarely been studied before. It is unclear how these approaches regarding duration and key elements may influence users' emotions, attention, memory, and attitudes.

This research compared the advertising effectiveness of mobile-feed video ads of different durations and layouts concerning their key elements. We conducted a betweengroup experiment involving 40 participants who were asked to view video ads from eight different brands while reading mobile feeds. We measured their attention, emotion, memory, and attitudes towards the video ads through various measures, including electroencephalography (EEG), eye tracking, self-report questionnaires, and interviews. We aimed to determine an appropriate duration and arrangement of the key elements for mobile-feed video ads.

This research offers three significant contributions. Firstly, we investigated the influence of duration and key elements on video ads' advertising effectiveness in mobile feeds. Secondly, we proposed a set of methods for studying advertising effectiveness in mobile feeds; specifically, we used EEG, eye tracking, self-report questionnaires, and interviews to compare advertising effectiveness. Thirdly, this research helps clarify some preferred features of video ads in mobile feeds. Moreover, it will also provide some useful suggestions for advertisers who design video ads.

\section{Related Works}

Mobile ads refer to ads that are promoted through mobile devices, such as mobile phones, tablets, or laptops. Unlike traditional ads like television or newspaper ads, mobile ads have enjoyed much more media richness and interactivity owing to the power of new technological devices [18]. Traditional ads are usually presented on a one-way medium, which means that users can only receive information from the medium; however, mobile ads involve a two-way interaction. Users can watch or read ads and immediately give feedback, like deleting the ads or clicking the ads. Consequently, the ad systems can collect users' preferences and deliver future ads accordingly through various algorithms $[7-9,19]$.

Mobile ads are divided by transmission software medium into four groups: mobile messaging ads, mobile web ads, mobile video ads, and mobile app ads [20]. Mobile messaging usually refers to the ads delivered through short message services or e-mails. Mobile web ads are pictures, banners, or links in mobile browsers. Mobile video ads are advertising videos that are played either before, after, or during videos. Both web ads and video ads can be embedded in mobile apps to improve interactivity [21]. In fact, web ads and video ads are ubiquitous in many mobile apps with information feeds.

In this research, we focused on video ads, which we defined as online display ads that have video within them. Some researchers have found that applying video ads in a video stream is very effective in web advertising [22, 23]. Viewers' motivations to watch video ads vary from person to person, and the ways they watch videos ads are closely related to their motivations [24]. Motivations such as social connectedness, relaxation, information, escapism, passing time, and entertainment encourage users to watch ads. In addition, some research [24] suggested that advertisers take advantage of the interactive and participatory nature of the web apps and develop more accurate ads according to the interactive characteristics of each media platform. Tradition video ads also have some disadvantages. Intrusiveness can prevent users from watching ads. On some occasions, ads can be very annoying since their forms and content are quite different from the content users are reading or viewing.

Native ads, on the other hand, can reduce intrusiveness when users view them [2-4]. Many researchers have indicated native ads' advantages over traditional mobile ads as the native ads have higher click-through rates [25-27]. Nevertheless, some researchers argued that the native ads could also lead to drastic drops in click-value rates because the disguised nature of native ads is likely to cause users' annoyance after they realize that they have been tricked $[28,29]$. Users are likely to feel deceived after clicking on an ad that they initially thought was an organic listing, particularly when the ads are presented in low serial positions [3]. However, these discussions were limited in the native ads in text, which need an extra click to read full contents. Few studies have looked into native video ads that are automatically played in the information feed without extra clicks.

Contrary to native ads in text, native video ads are usually perceived as less deceptive. It does not interrupt the user experience with unnecessary extra clicks, meaning the content is more likely to be engaged with rather than an annoying distraction in their feed [30]. Given that users usually scan the native video ads when rolling down the feeds, it is particularly crucial for advertisers to rapidly grab users' attention and deliver core contents of the video ads within a limited time. To date, most studies on native advertising $[3,12,13]$ have been focused on how users' recognition of native ads affects their evaluations of the ads and advertiser [28, 29]. Little work has been devoted to native video ads' design principles, especially for the duration and key elements. This work is essential for practitioners in the advertising industry to design better video ads of high advertising effectiveness without damaging users' experience. It will also shed light on future research regarding native video ads' design in the mobile information feed. 
Duration is a key factor that influences advertising effectiveness. Many researchers found that viewers' memories of the ads are strongly related to their exposure time to the ads $[16,31,32]$. The longer viewers exposed themselves to the ads, the more possibilities they remembered the contents. Li and Lo [33] conducted an empirical study on the effect of duration and position of the in-stream video ads. It was found that longer duration and midroll ads lead to better brand recognition. Goodrich et al. [16] also found that longer preroll ads were perceived as less intrusive than the shorter ads. However, longer ads do not always produce better results. Krishnan and Sitaraman [22] conducted a quasiexperiment and found that longer ads were less likely to be watched to completion than were shorter ads. Ads placed in the middle of a video were more likely to be watched to completion than those placed at the beginning or end, which was also verified by $\mathrm{Li}$ and Lo [33]. One possible explanation of this phenomenon is that longer ads are more intrusive and cause users' avoidance of the ads [16]. Advertising avoidance refers to "actions that media users take to reduce exposure to an ad's content" [34]. Fan et al. [6] found that perceived goal impediment and perceived ad clutter contributed to the advertising avoidance. It is worth noting that the users were more pleasant if they were provided with a "skip" option in the ads [22] because it increased participants' attention on the ads and reduced perceived intrusiveness. Therefore, we can imply that longer video ads in mobile feeds, which interrupt users' reading flow more, are more likely to be intrusive than shorter videos ads, thereby producing more unpleasant emotion.

Given that short videos cannot provide enough information, key elements (logo and text) are introduced into the video ads to compensate for the shortcoming. Previous research [35-39] found that logo elements and text elements played an important role in capturing users' attention. Pieters and Wedel [38] found that the text element captures attention in proportion to its size, and the brand element transfers attention to the other elements effectively. Viswanathan et al. [39] also found that a pictorial logo would help users remember and understand more of the ad contents. Li et al. [37] found that text in tourism photographs drew the most visual attention regardless of the text language. They also confirmed that participants are more likely to be influenced by texts in the pictorial ads. Thus, we can imply that users perceived more advertising effectiveness towards the ads with key elements than the ads without key elements in mobile feeds.

\section{Materials and Methods}

3.1. Participants. We recruited 40 participants $(27$ men and 13 women) through a social network on WeChat. Participants were aged from 20 to 43 years $(M=29.33$ years, $\mathrm{SD}=6.67)$. Half were aged $<30$ years. Two-thirds $(67.5 \%)$ were either students or employees of the authors' university. Sixty percent used their mobile phones for more than three hours per day. Nearly all (92.5\%) admitted that they used social apps daily, which was followed by news apps (55\%) and shopping apps (55\%) in prevalence. All participants were right-handed to avoid individual differences in the dominant hemisphere [40].

3.2. Experiment Design and Stimuli. The experiment was conducted with four groups, in which participants viewed four different types of video ads. These ads differed in the duration and arrangement of their key elements. Each participant was asked to finish eight experiment tasks and view eight different ads from eight different brands. In each task, participants were required to read a series of news feeds, and the ads appeared in the feeds as they scrolled down the screen. The ads were played automatically without any sound. The appearance of the native video ads was similar to the news in the feed, but they were not relevant in content.

There were two independent variables in this research: duration and presence of the key elements. The variable duration had three levels: 6 seconds, 15 seconds, and 30 seconds. Six-second ads were explicitly designed for in-feed advertising, allowing users to view the entire ad contents in a very short time, whereas 15 - and 30 -second ads are more typical of TV or video website advertisements. Second, key elements refer to logos and text in the video ads, which had two levels: with and without key elements (Figure 1).

Four types of video ads were used in the experiment: 6second ads with key elements (M6), 15-second ads with key elements (M15), 15-second ads without key elements (U15), and 30-second ads without key element (U30). Except for the duration and key elements, the four versions were similar in content. Although our experiment utilized four groups, it was not a standard orthogonal experimental design. Certain types of ads (6-second ads without key elements and 30second ads with key elements) were unavailable. It is one of the limitations of this research; however, there is still value to our between-group comparisons.

The ads were provided by a Chinese Internet company that owns one of the most popular news aggregation apps in China. The content comprised a car ad, a milk powder ad, a cosmetics ad, an e-commerce ad, a thermos ad, a mineral water ad, and two mobile phone ads.

3.3. Measurement. Venkatraman et al. [41] proposed a framework to assess advertising effectiveness. It includes four core aspects: attention, emotions, memory, and attitudes. Attention, which refers to the ability to attract users' focus on an ad, is usually measured through participants' self-reports. However, self-reports cannot distinguish endogenous attention from exogenous attention; therefore, objective methods like eye tracking are usually applied in these situations. Our experiment used eye tracking to measure user's attention while they read their news feed. Emotion is defined as the outward expression of emotion and can be classified into two dimensions: valence and arousal, and neurophysiological methods are recommended to measure participants' emotions. In the experiment, we also implemented the Self-Assessment Manikin Scale [42] to measure participants' emotions and affection. Memory in advertising research is usually focused on information 


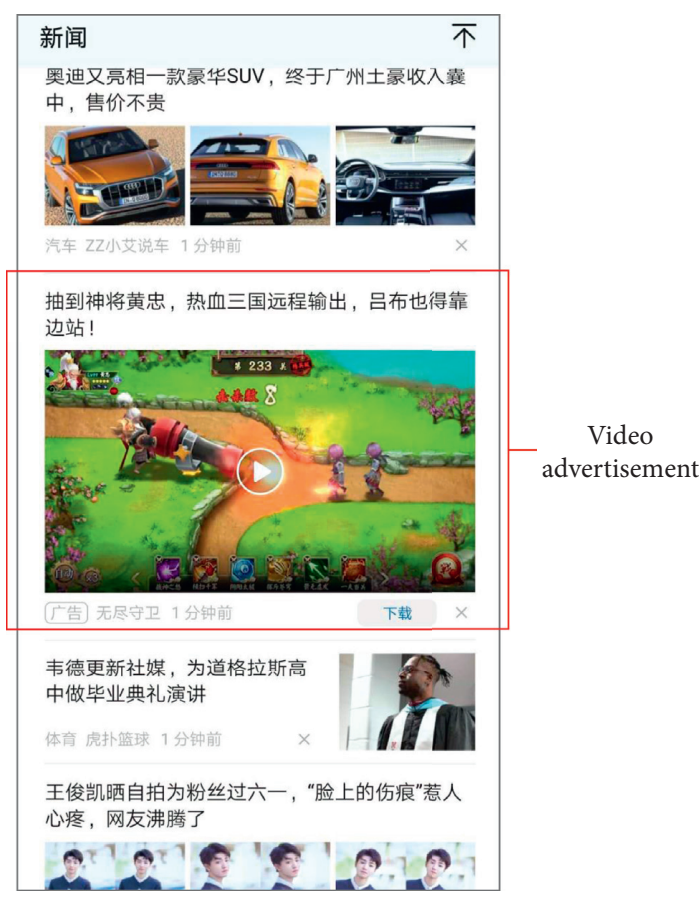

(a)

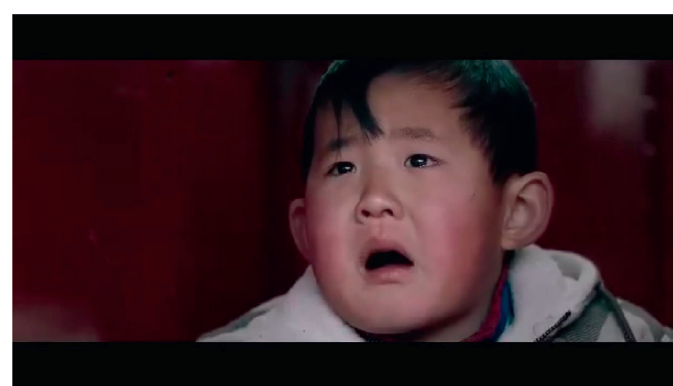

Screenshot of a video ad without key element

(b)

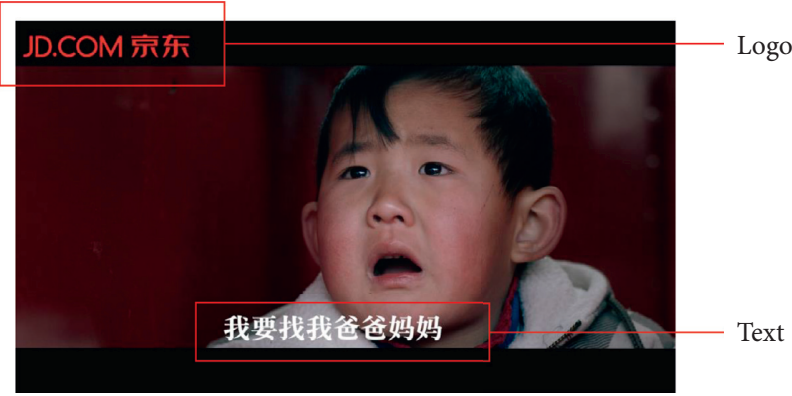

Screenshot of a video ad with key elements

(c)

FIGURE 1: Illustrations of a video advertisement (ad) in mobile feeds (a), a video ad without key element (b), and a video ad with key elements (c).

retrieval. More specifically, two aspects are considered in the measure of memory: recall and recognition [43]. We used a questionnaire and interview to measures participants' memory of the ads presented in the news feed. Lastly, attitudes refer to people's attitudes and purchase intentions to the products featured in the ads. Venkatraman argued that attitudes are likely to be influenced by people's preimpression of the brand, and he suggested that applying neurophysiology would be a promising direction to measure attitudes. However, it was not practical to measure participants' emotions and attitudes through neurophysiology simultaneously in the experiment. We used a questionnaire and interview to measure participants' attitudes.

3.3.1. Eye Tracking. Eye tracking has been used in ample research related to advertising in the past $[35,37,38]$. One of the advantages of eye tracking is that it can provide a dynamic trace of a person's attention [44]. Other eye movements, such as fixation, can also indicate a person's information processes. In our research, we considered three indicators to describe users' eye tracking process in different ads: percentage of dwell time in the area (PDT), first fixation time (FFT), and average fixation time (AFT). PDT refers to the percentage of time that participants spend viewing the area of interest (AOI) in the total advertising time. FFT refers to the first-time participants' sight stays at the AOI. AFT refers to the average time that participants' fixations fall in the AOI. We divided the infeed video ads into different AOIs according to the ad types. For nonoptimized ads, there were three AOIs, including the whole ad area, the title area, and the video area. The tile area shows the basic information about this video or a slogan. For mobile-optimized ads, a logo area and a text area were always shown in the ads. It should be noted that the positions of the texts and the logos were not fixed in the video. The layouts of the video ads in the news feed are shown in Figure 2. 


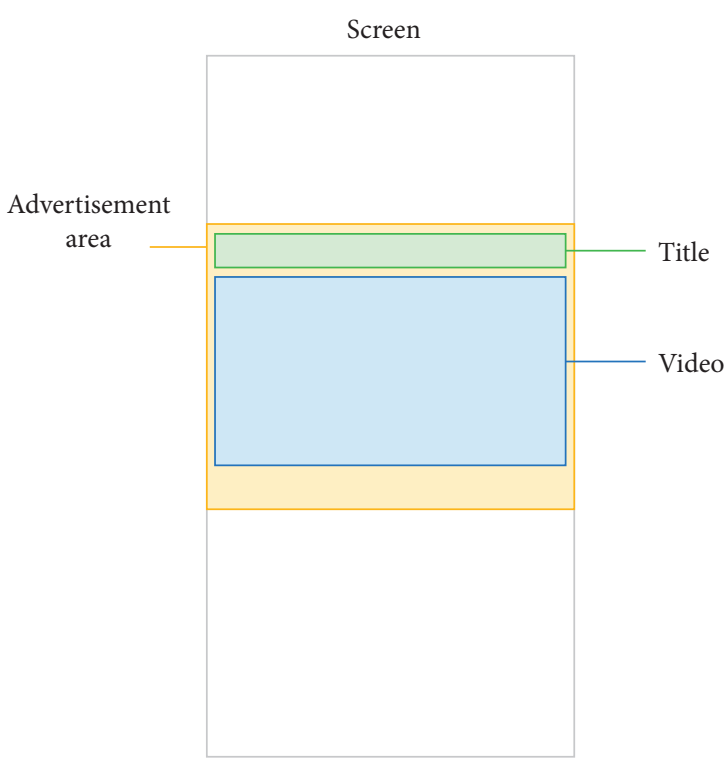

(a)

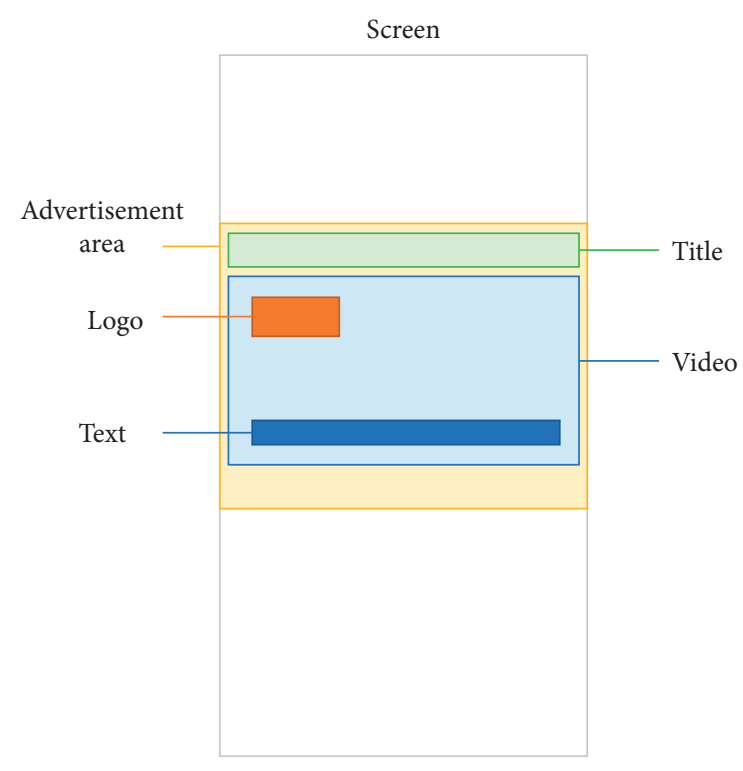

(b)

Figure 2: Layout of area of interest (AOI) in ads without key elements (a) and layout of AOI in ads with key elements (b).

The eye movement tracking device used in the experiment was the SMI ETG2W glasses eye tracking system. The sampling frequency of this device was $60 \mathrm{~Hz}$ for both eyes, and the tracking resolution was $0.1^{\circ}$. The gaze positioning accuracy was $0.5^{\circ}$, and the tracking range was horizontal $80^{\circ}$ and vertical $60^{\circ}$. The glasses HD lens resolution was $1280 * 960$. The field of view was horizontal $60^{\circ}$ and vertical $45^{\circ}$. The weight of the glasses was $47 \mathrm{~g}$. Participants were asked to put their chins on a supporter to reduce the effect of head movement. The experimental platform is shown in Figure 3.

3.3.2. EEG. In past research, EEG was shown to be a practical method to measure emotions [45]. Frontal EEG asymmetry served as a moderator to indicate participants' valence and arousal in our research. We used an Emotiv EPOC neuroheadset to collect signals with a $128 \mathrm{~Hz}$ sample rate in 14 channels $(A F 3, F 7, F 3, F C 5, T 7, P 7, O 1, O 2, P 8, T 8$, $F C 6, F 4, F 8$, and AF4). To reduce the effect of participants' movement when they were using mobile phones, we first asked participants to view the feed for 3 minutes to record their base signal.

We measured the valence of emotion by comparing the asymmetry of the power spectral density in the left and right frontal lobes. More specifically, we used the differential value between $F 3$ and $F 4$ in the $\alpha$ band as a valence indicator [46]:

$$
\text { valence }=\frac{\alpha_{F 4}}{\beta_{F 4}}-\frac{\alpha_{F 3}}{\beta_{F 3}} \text {. }
$$

It should be noted that a higher value of valence indicates more positive emotions; however, the sign of the value (positive or negative) does not mean the emotion is positive or negative valence.

In addition, arousal was related to high beta power and low alpha activity in the parietal lobe [46]. The beta band is usually related to alertness and excitement, and the alpha band is typically related to relaxation. It is common practice to use the alpha/beta ratio as an indicator of a person's arousal:

$$
\text { arousal }=\frac{\alpha_{F 3}+\alpha_{F 4}+\alpha_{A F 3}+\alpha_{A F 4}}{\beta_{F 3}+\beta_{F 4}+\beta_{A F 3}+\beta_{A F 4}} .
$$

3.3.3. Questionnaires and Interview. Attitude towards ads was measured through a questionnaire. The questionnaire was adapted from Hsiao and Chang's research [47]. Hsiao and Chang divide consumers' attitudes toward ads into six dimensions: continuance intention, satisfaction, perceived usefulness, confirmation, perceived value, and perceived trust. Before the experiment, we measured participants' initial attitudes to reduce the effect of individual differences toward ads. In the following analysis, we include the initial attitude in the statistical model as a covariate.

After participants viewed each ad, they were asked to report their attitude concerning three aspects: attitude toward the ad itself, attitude toward the brand, and the product featured in the ad. Further, participants' desirability was measured in the questionnaires by asking their intention to buy or recommend the products and brands. Questions were responded to using 7-point Likert scales. Valence and arousal were measured through the Self-Assessment Manikin Scale [48]. The questionnaire used a pictorial 9-point instrument to measure participants' valence and arousal. Although there are three emotion dimensions in Hodes, Cook Iii, and Lang's model, dominance was not included in the model of [41].

We measured participants' memory through interviews. After participants viewed each ad, an experimenter first asked participants to describe the brands and products. Then, participants were required to recall the ad content or 


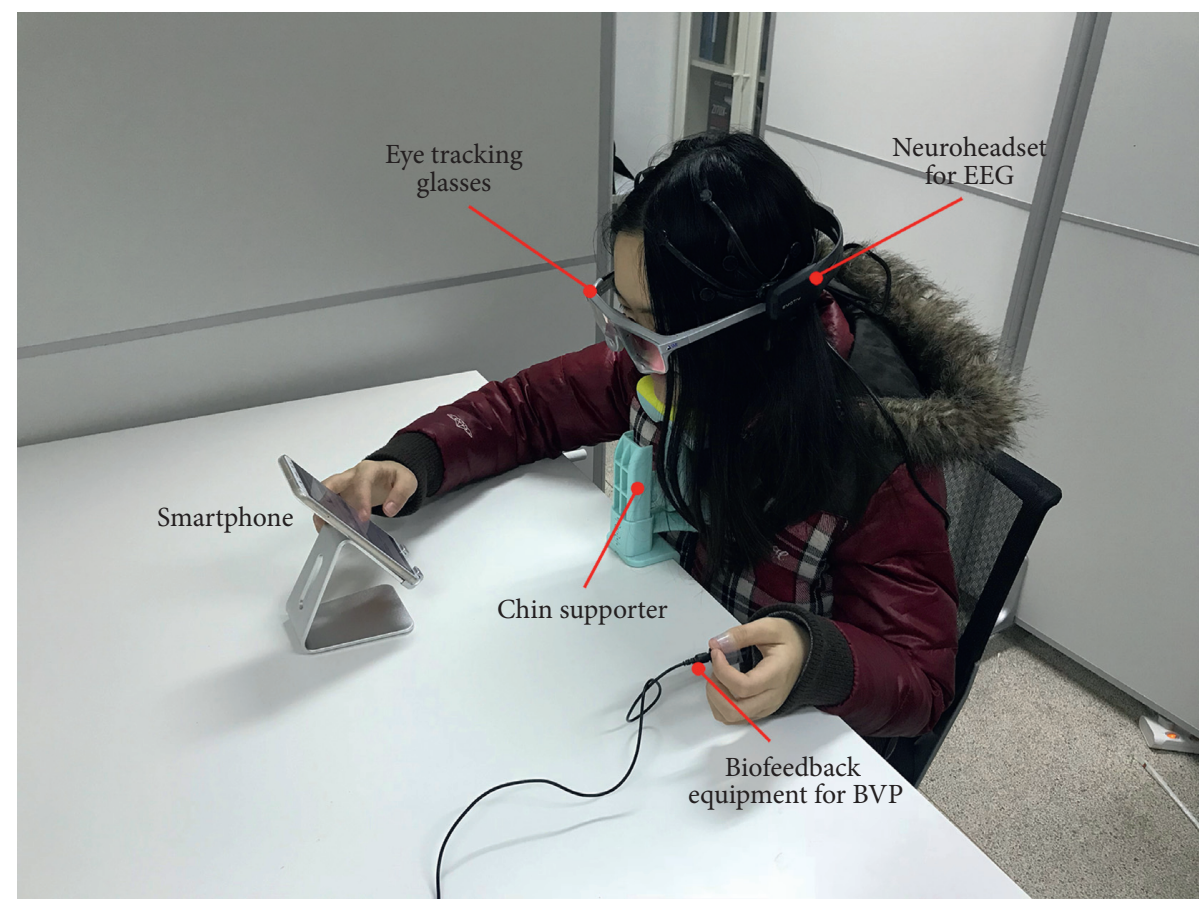

FIgURE 3: Experiment platform.

plot, which were audiorecorded. After that, the experimenter asked participants about the most impressive scenes or words in the ad. At the end of the interview, participants expressed their feelings about the ad freely.

3.4. Procedure. Participants in each group independently viewed eight ads in a news feed on a smartphone. Each ad could be regarded as a small section. In each section, the video ads appeared in the middle of the feed, and participants were supposed to read the news they like and view the ads. After they finished watching the ads, they could continue to read the rest of the news. The reason we chose news as opposed to a social feed was to hinder the influence of participants' emotions.

The entire experiment proceeded as follows: (1) participants read and signed the informed consent form; then, they completed the preexperimental questionnaire including their personal information, ads preference, and their attitude toward ads; (2) the experimenter introduced the experimental task to participants; (3) the experimenter helped participants put on their EEG neuroheadset and eye tracking devices; (4) in each section, participants read the feed and watched the ads; then, they completed a questionnaire and were interviewed; and (5) after participants finished the eight sections, the experimenter helped participants take off the measurement equipment.

\section{Results and Analysis}

4.1. Eye Tracking. We used three indicators to measure participants' attention when they were watching the ads. The four experimental groups significantly differed per the three indicators (Table 1) according to the analysis of variance (ANOVA).
TABLe 1: Mean values, standard deviations, and the analysis of variance results concerning the eye movement measure.

\begin{tabular}{cccccccc}
\hline & & M6 & M15 & U15 & U30 & F & $p$ \\
\hline \multirow{2}{*}{ PDT } & Mean & 84.88 & 88.13 & 88.84 & 84.01 & \multirow{2}{*}{3.32} & \multirow{2}{*}{0.02} \\
& SD & 12.53 & 9.66 & 9.78 & 12.99 & & \\
\hline \multirow{2}{*}{ AFT } & Mean & 239.77 & 263.75 & 339.33 & 268.34 & \multirow{2}{*}{28.60} & $<0.01$ \\
& SD & 54.32 & 49.76 & 105.09 & 51.06 & & \\
\multirow{2}{*}{ FFT } & Mean & 198.10 & 207.60 & 244.13 & 193.52 & \multirow{2}{*}{2.21} & \multirow{2}{*}{0.087} \\
& SD & 132.69 & 114.36 & 162.14 & 123.11 & & \\
\hline
\end{tabular}

$\mathrm{PDT}=$ percentage of dwell time in the area, $\mathrm{FFT}=$ first fixation time, and $\mathrm{AFT}=$ average fixation time

For PDT, there was a significant difference between the four groups $(p=0.02)$. Tukey's post hoc test revealed that the M15 and U15 group's mean scores were higher than the U30 group. For AFT, there was also a significant difference between the four groups $(p<0.01)$. Tukey's post hoc test revealed that the U15 group's mean score was significantly higher than the other three groups' scores, and the U30 group's score was higher than that of the M6 group. Lastly, for FFT, there was a marginally significant difference between the four groups $(p=0.09)$. Tukey's post hoc test revealed that the U15 group's mean score was significantly higher than the U30 group's score.

Regarding key elements, we compared the PDT, AFT, and FFT between the three key elements (title, text, and logo) in the M6 group and M15 group by two-way ANOVA. The results revealed main effect of key elements in terms of PDT $(p<0.01)$ , AFT $(p<0.01)$, and FFT $(p=0.02)$. Post hoc analysis indicated that the text area enjoys more attention from the participants since the PDT, AFT, and FFT are higher than the other two areas. The results also showed the interaction effect between duration and key elements $(p<0.01)$ in PDT 
(Figure 4). Post hoc analysis indicates that participants spent much more dwell time on the text area when viewing long duration video ads. In contrast, participants spent more time on the title area when viewing the short duration videos. No other interaction effects were found.

4.2. EEG. We used EEG to measure participants' valence and arousal when they were watching the ads. We conducted an ANOVA to compare the four different ad groups (Table 2). There was a significant difference $(p<0.01)$ in valence. Tukey's post hoc test revealed that the M6 and U30 groups' valence scores were significantly more positive compared to the other two groups. There was also a significant difference regarding arousal $(p<0.01)$. Tukey's post hoc test revealed a significant difference between the M6 group and the other three groups. The mean values of the EEG for emotional valence and arousal for the four ads groups are shown in Figure 5, which implies that M6 was more favored by participants than the other three groups since higher valence and arousal usually lead to better advertising effectiveness.

4.3. Self-Report Questionnaires. We measured participants' attitudes, emotions, and intentions through a self-report questionnaire with an ANOVA (Table 3). There was a significant difference in participants' self-report valence $(p<0.01)$, and Tukey's post hoc test revealed that the valence of the M6 group was more positive as compared to the U15 group (smaller values indicate more positive valence), which was consistent with the EEG result. There was also a significant difference in participants' attitudes toward the brands, and Tukey's post hoc test revealed that the U30 group's score was significantly higher than the M6 group's score.

We also investigated the correlation among participants' background characteristics (age, sex, consumption level, and phone usage time per day) and the questionnaire measures above. The results indicated that participants' intentions to use, purchase, and recommend were significantly related to their valence and brand attitude; although, no significant differences were found concerning these measures in the ANOVA described above (Figure 6).

4.4. Interviews. We interviewed participants after each section to measure their memories of the ads and their attitudes. We encouraged participants to recall as much ad content as possible, and the level of detail they described indicated their memory of the ads. We encoded the interview results through NVivo, qualitative data analysis software, and divided their descriptions into four categories: wrong description, obscure description, brief description, and detailed description. We divided their attitudes into three categories per their responses.

The results of the Kruskal-Wallis test are shown in Table 4 . There was a significant difference between the four groups regarding detailed description $(p<0.01)$. Pairwise comparisons revealed that the number of participants in the U30 group who described the ads in detail was significantly

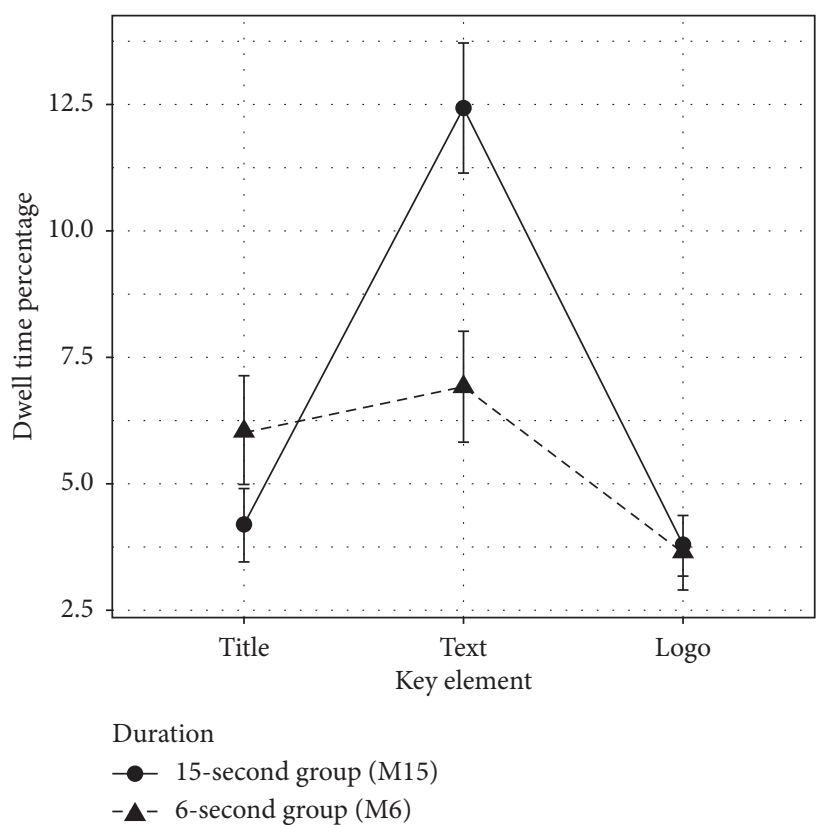

FIGURE 4: Interaction plot of key elements and duration.

TABLE 2: Mean values, standard deviations, and analysis of variance results concerning electroencephalography.

\begin{tabular}{lccccccc}
\hline & & M6 & M15 & U15 & U30 & F & $p$ \\
\hline \multirow{2}{*}{ Valence } & Mean & 3.84 & 0.71 & -0.65 & 3.73 & \multirow{2}{*}{10.92} & $<0.01$ \\
& SD & 5.51 & 3.84 & 7.87 & 6.38 & & \\
\hline \multirow{2}{*}{ Arousal } & Mean & 1.74 & -1.31 & -1.63 & -2.93 & \multirow{2}{*}{13.41} & $<0.01$ \\
& SD & 6.73 & 2.99 & 4.83 & 3.94 & & \\
\hline
\end{tabular}

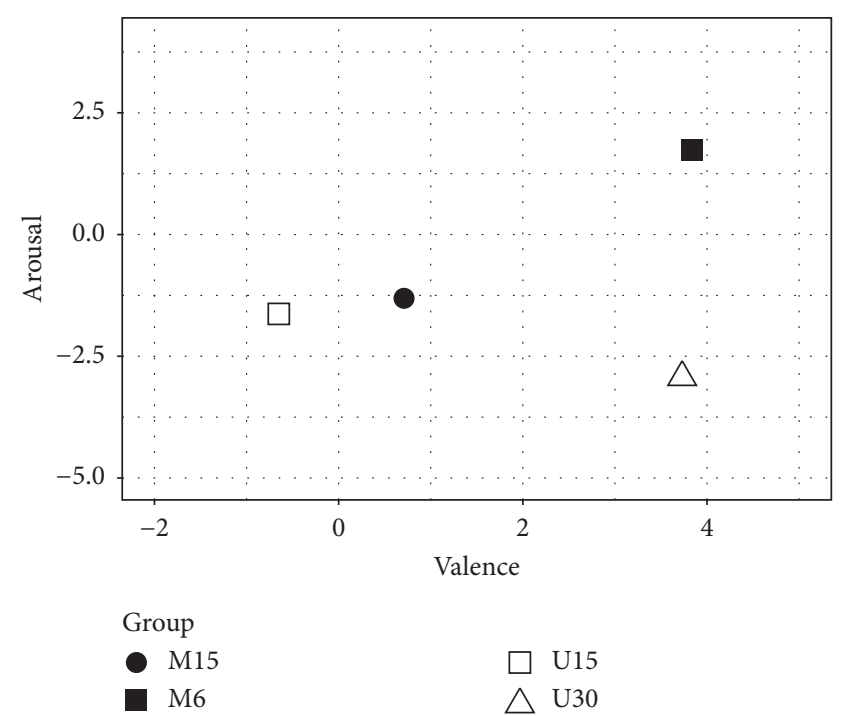

FIgURE 5: The mean values of the EEG for emotional valence and arousal for the four advertisement groups.

larger than the other three groups, which implied that participants in the U30 group have a better memory of the ads than the other three groups. Although there was no 
TABLE 3: Mean values, standard deviations, and analysis of variance results concerning the questionnaires.

\begin{tabular}{|c|c|c|c|c|c|c|c|}
\hline & & M6 & M15 & U15 & U30 & $\mathrm{F}$ & $p$ \\
\hline \multirow{2}{*}{ Valence } & Mean & 3.43 & 3.89 & 4.40 & 3.93 & \multirow{2}{*}{4.70} & \multirow{2}{*}{$<0.01$} \\
\hline & SD & 1.75 & 1.78 & 1.70 & 1.45 & & \\
\hline \multirow{2}{*}{ Arousal } & Mean & 5.43 & 5.05 & 5.25 & 5.36 & \multirow{2}{*}{0.77} & \multirow{2}{*}{0.51} \\
\hline & SD & 2.05 & 1.83 & 1.63 & 1.53 & & \\
\hline \multirow{2}{*}{ Attitude toward the ad } & Mean & 4.23 & 4.34 & 4.15 & 4.40 & \multirow{2}{*}{0.65} & \multirow{2}{*}{0.58} \\
\hline & SD & 1.35 & 1.32 & 1.42 & 1.29 & & \\
\hline \multirow{2}{*}{ Attitude toward the brand } & Mean & 4.54 & 4.88 & 4.73 & 5.09 & \multirow{2}{*}{2.69} & \multirow{2}{*}{0.05} \\
\hline & $\mathrm{SD}$ & 1.42 & 1.25 & 1.29 & 1.00 & & \\
\hline \multirow{2}{*}{ Attitude toward the product } & Mean & 4.42 & 4.61 & 4.31 & 4.63 & \multirow{2}{*}{1.47} & \multirow{2}{*}{0.22} \\
\hline & $\mathrm{SD}$ & 1.15 & 1.03 & 1.29 & 1.16 & & \\
\hline \multirow{2}{*}{ Intention to use } & Mean & 3.89 & 4.35 & 4.21 & 3.90 & \multirow{2}{*}{0.88} & \multirow{2}{*}{0.45} \\
\hline & SD & 1.71 & 1.81 & 1.96 & 1.69 & & \\
\hline \multirow{2}{*}{ Intention to purchase } & Mean & 3.83 & 4.29 & 4.21 & 3.76 & \multirow{2}{*}{1.22} & \multirow{2}{*}{0.30} \\
\hline & SD & 1.75 & 1.79 & 1.97 & 1.72 & & \\
\hline \multirow{2}{*}{ Intention to recommend } & Mean & 3.99 & 4.17 & 4.06 & 3.68 & \multirow{2}{*}{0.78} & \multirow{2}{*}{0.51} \\
\hline & SD & 1.57 & 1.75 & 1.88 & 1.52 & & \\
\hline \multirow{2}{*}{ General attitude } & Mean & 4.40 & 4.61 & 4.40 & 4.71 & \multirow{2}{*}{0.88} & \multirow{2}{*}{0.45} \\
\hline & $\mathrm{SD}$ & 1.19 & 1.07 & 1.23 & 0.96 & & \\
\hline
\end{tabular}

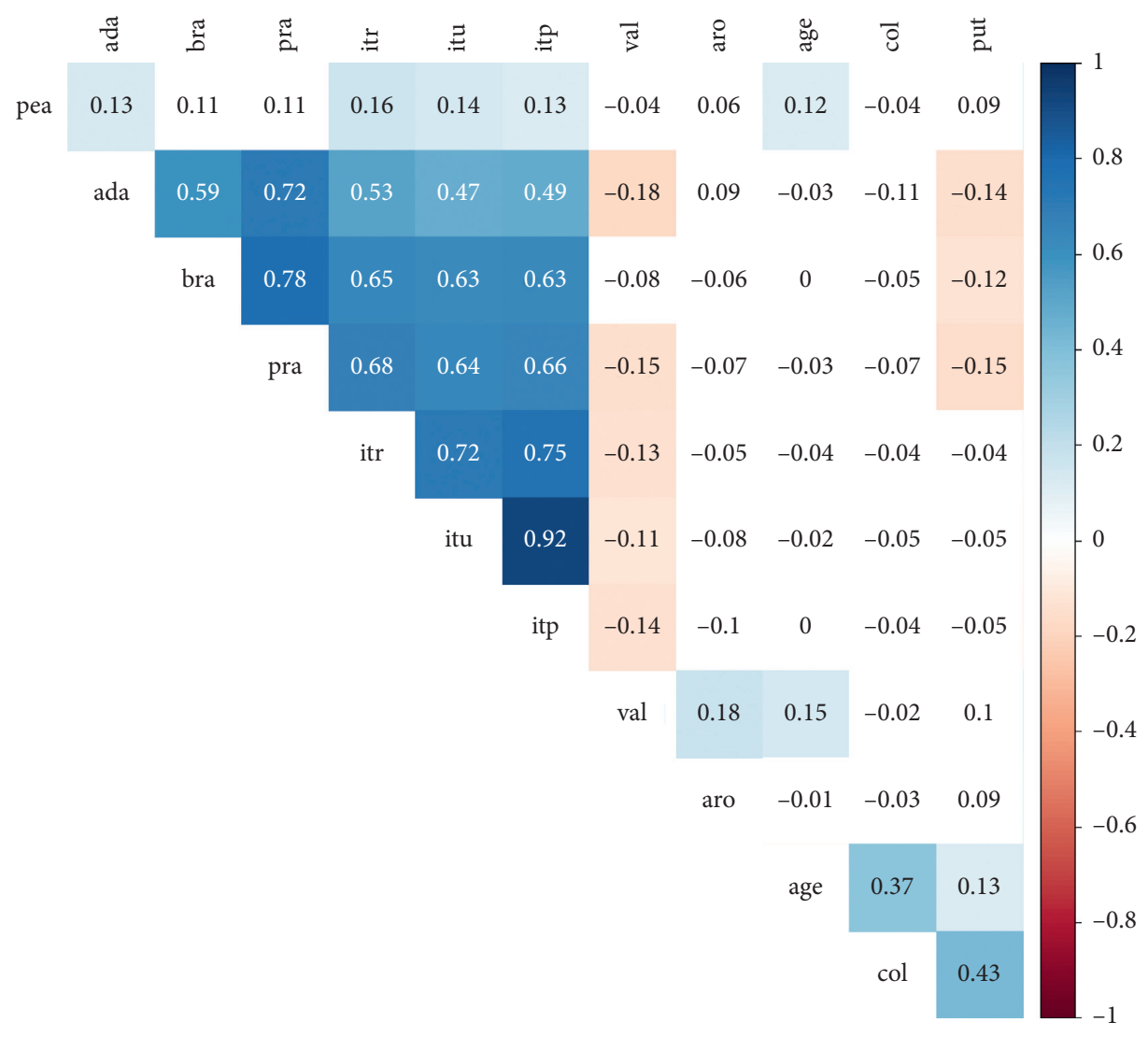

$$
\begin{array}{lll}
\text { pea }=\text { pre-experiment attitude } & \text { itr }=\text { intention to recommend } & \text { aro }=\text { arousal } \\
\text { ada }=\text { advertisement attitude } & \text { itu }=\text { intention to use } & \text { age }=\text { age } \\
\text { bra }=\text { brand attitude } & \text { itp }=\text { intention to purchase } & \text { col }=\text { consumption level } \\
\text { pra }=\text { product attitude } & \text { val }=\text { valence } &
\end{array}
$$

Figure 6: Correlation among the participants' background characteristics and questionnaire measures. The blue color indicates a significant positive correlation $(p<0.05)$, whereas the red color indicates a significant negative correlation $(p<0.05)$. 
TABLE 4: Mean values, standard deviations, and Kruskal-Wallis test results concerning the interview.

\begin{tabular}{lccccccc}
\hline & & M6 & M15 & U15 & U30 & $\chi^{2}$ & $p$ \\
\hline \multirow{2}{*}{ Wrong description } & Mean & 0.63 & 0.13 & 0.75 & 0.50 & \multirow{2}{*}{4.19} & \multirow{2}{*}{0.24} \\
& SD & 1.11 & 0.33 & 0.66 & 0.50 & & \\
\hline \multirow{2}{*}{ Obscure description } & Mean & 1.38 & 0.63 & 1.00 & 0.50 & \multirow{2}{*}{2.76} & \multirow{2}{*}{0.43} \\
& SD & 1.11 & 0.48 & 1.32 & 0.50 & & \\
\hline \multirow{2}{*}{ Brief description } & Mean & 6.88 & 6.75 & 5.88 & 4.50 & \multirow{2}{*}{6.13} & \multirow{2}{*}{0.11} \\
& SD & 2.15 & 1.09 & 1.36 & 1.87 & & \\
\hline \multirow{2}{*}{ Detailed description } & Mean & 1.13 & 2.50 & 2.25 & 4.50 & \multirow{2}{*}{15.00} & \multirow{2}{*}{$<0.01$} \\
& SD & 0.93 & 0.87 & 1.09 & 1.80 & & \\
\multirow{2}{*}{ Positive attitude } & Mean & 5.50 & 5.63 & 5.75 & 5.75 & \multirow{2}{*}{6.23} & \multirow{2}{*}{0.72} \\
& SD & 1.32 & 3.16 & 1.79 & 1.85 & & \\
\hline \multirow{2}{*}{ Neutral attitude } & Mean & 4.38 & 3.13 & 2.50 & 3.88 & \multirow{2}{*}{5.38} & \multirow{2}{*}{0.61} \\
& SD & 1.32 & 2.37 & 1.80 & 1.27 & & \\
\hline \multirow{2}{*}{ Negative attitude } & Mean & 0.75 & 2.38 & 3.25 & 3.25 & \multirow{2}{*}{6.71} & \multirow{2}{*}{0.08} \\
& SD & 1.09 & 0.86 & 1.30 & 1.48 & & \\
\hline
\end{tabular}

significant difference regarding wrong descriptions, pairwise comparisons revealed that the descriptions of participants from the U15 group were more likely to be wrong than the M15 group. The result implies that the ads with key elements enhanced the participants' memory of the ads. There was also a marginal difference between the four groups in negative attitudes $(p<0.01)$. Pairwise comparisons revealed that participants in the M6 group produced fewer negative attitudes than the other three groups.

\section{Discussion}

5.1. Attention. Attention was measured through eye tracking in our research. We used three measures to describe participants' attention when they were watching ads. We found that participants' sight stayed in the ad area relatively longer in the M15 and U15 groups than the U30 group because the participants were likely to get distracted when viewing mobile feeds when the ads exceeded a certain length. No significant differences were found between the U15 and U6 groups, which implies that 15 seconds is an appropriate length for mobile-feed ads before users become distracted. The AFT for U15 and U30 groups was longer than the AFT of the M15 and M6 groups, which is because the videos for the latter had more elements per second than the videos in the former. Further, participants' sight jumped between the key elements (logos and texts) and video contents. There were fewer elements in the videos in unoptimized group videos; therefore, participants' sight was relatively more fixed. Moreover, the U30 group's FFT was longer than that of the other three groups, which implies that participants tend to fix their eyes longer in U30 videos. Therefore, when viewing the mobile-optimized ad, participants received more information. Their attention was relatively scattered compared to other conditions; on the other hand, participants' sight was comparatively more fixed when viewing unoptimized videos.

We then compared participants' attention in the three different key elements - title, text, and logo area. There was an interaction effect between the groups and key elements.
Since we only compared the key elements in the M6 group and M15 group, which differed in duration, the interaction effect is, as a fact, between duration and key elements. Therefore, we found that participants would pay more attention to the ad title area when the ad duration was short; however, as the ad duration increased, participants shifted their attention to the text areas. This implies that ad titles play a key role when the ad duration is very short, and texts are vital when the ad duration becomes longer. It would be efficient for advertisers to design a good title for short ads, and it would be better to focus on the texts in longer ads.

5.2. Emotions. In this research, we evaluated participants' emotions from two dimensions: valence and arousal. Three methods were used to measure these two dimensions: EEGs, questionnaires, and interviews, and the results of these three methods were consistent. The EEG results indicated that the M6 and U30 groups were more positive than the other two groups; however, the M6 group had higher arousal than did the U30 group. The questionnaire results revealed that the M6 group was more positive than was the U15 group. The interview results revealed that participants held less negative attitudes in the M6 and M15 groups than the other two groups, which also reflects a more positive valence in emotion. Thus, the M6 group's ads were the most preferred by the participants, possibly because shorter ads least interrupt the users reading flow and thereby induced less intrusiveness. On the contrary, a long duration ad will interrupt the reading process, affecting users' experience of the mobile feeds. Watching a long ad in mobile feeds can also cause fatigue and boredom; therefore, it is better to use a shorter duration video ad in mobile feeds to reduce negative emotions. It is also worth noting that we compared relative positive and negative emotions rather than absolute positive and negative values. Therefore, the above comparison does not explain whether emotions were positive or negative when viewing a certain group of ads.

5.3. Memory and Attitude. Memory was mainly analyzed through the results of the interviews. By encoding the interview results, we can obtain participants' memories of the ad content. We found that participants in the M6 group recalled less of the advertising content, and participants in the U30 group remembered more details about the ads than the other groups. This result is similar to what we found concerning attitude, which was measured with the questionnaires. The results indicated that participants' attitudes toward the brand were higher in the U30 group than in the M6 group. Although no other significant differences were found, the correlation analysis revealed that participants' intention to use, purchase, and recommend was significantly related to valence and brand attitude.

The reason why the U30 group displayed better memory and a positive brand attitude than did the M6 group was that the ads in the U30 group had enough time to advertise the products to participants. Participants have more of a chance to learn about the product and form opinions in longer ads than shorter ads, which may foster positive brand attitudes. 
Another interesting finding was that participants of the U15 group made more mistakes when they were asked to recall the content of the ads compared to the participant of the M15 group, possibly because the key elements in M15 helped users to capture the key information about the ads. The result implied that adding key elements in the mobilefeed video ads would facilitate a better understanding of the ads.

5.4. Limitations and Future Directions. Our study does face several limitations. Firstly, the number of our participants was relatively small. However, given that each participant was asked to view eight video ads from eight different advertisers separately, the total number of experiment trials is quite considerable. This could partially compensate for the deviation caused by an insufficient number of people. Secondly, the experiment was not orthogonally designed since two certain groups, 6-second ads without key elements group and 30-second ads with key element group, were not examined in our research. Despite this, we still found interesting and meaningful results regarding duration and key elements in mobile-feed video ads. The result can also help ad companies understand some preferred features of video ads in mobile feeds. Thirdly, we only considered one particular layout in the experiment. It was still unclear what influence other layouts might have on the effectiveness of mobile-feed video ads. Finally, the cultural difference might play a role in this research since all the participants were from China. Future research could explore the influence of cultural background on users' responses to mobile-feed video ads.

\section{Conclusions}

Our research investigated the effects of duration and key elements on the advertising effectiveness of video ads in mobile feeds through an empirical study. Specifically, we compared the effects of the ads of different duration (6 seconds, 15 seconds, and 30 seconds) and the presence of key elements on users' emotions, attention, memory, and attitudes in mobile native video ads. We also distinguished the role of different key elements (titles, text, and logos) in the ads of different durations. We recruited 40 participants and assigned them randomly into four groups to view four different types of ads in the news feed: 6-second ads with key elements, 15-second ads with key elements, 15-second ads without key elements, and 30-second ads without key elements. The results indicated that mobile video ads of short duration led to significantly higher valence and arousal, fostering positive emotions among mobile users. However, short duration ads made it difficult for users to capture key information about the ads, thus leading to poor understanding and memory of the ads. To compensate for the disadvantage, it was suggested to implement certain key elements, such as titles and text, to attract users' attention and help them get better comprehension in short video ads. In other words, short duration video ads with key elements were more preferred in mobile feeds. Per the key elements, titles were more effective in delivering information to users in the short video ads, whereas text played a more important role in native video ads of long duration. In summary, the present study fills a gap in the literature of native video ads in mobile feeds by exploring the effect of duration and key element design. In addition, our research has proposed a set of methods for studying advertising effectiveness in mobile feeds. The findings and methodology in our study will benefit practitioners in the advertising industry.

\section{Data Availability}

The raw/processed data required to reproduce these findings cannot be shared at this time as the data also form part of an ongoing study.

\section{Consent}

An informed consent form was signed by the participant shown in Figure 3 confirming that they agree to the use of their photographs in this article.

\section{Conflicts of Interest}

The authors declare that there are no conflicts of interest.

\section{Acknowledgments}

This research was supported by the Beijing Byte Dance Technology Co., Ltd.

\section{References}

[1] G. Fulgoni and A. Lipsman, "Digital game changers," Journal of Advertising Research, vol. 54, no. 1, pp. 11-16, 2014.

[2] C. Campbell and L. J. Marks, "Good native advertising isn't a secret,” Business Horizons, vol. 58, no. 6, pp. 599-606, 2015.

[3] P. Wang, G. Xiong, and J. Yang, "Serial position effects on native advertising effectiveness: differential results across publisher and advertiser metrics," Journal of Marketing, vol. 83, no. 2, pp. 82-97, 2019.

[4] S. Krouwer, K. Poels, and S. Paulussen, "Exploring readers' evaluations of native advertisements in a mobile news app," Journal of Media Business Studies, vol. 16, no. 2, pp. 77-94, 2019.

[5] E. Mao and J. Zhang, "What drives consumers to click on social media ads? The roles of content, media, and individual factors," in 2015 48th Hawaii International Conference on System Sciences, pp. 3405-3413, Kauai, HI, USA, January 2015.

[6] S. Fan, Y. Lu, and S. Gupta, "Social media in-feed advertising: the impacts of consistency and sociability on ad avoidance," in Proceedings of Twenty First Pacific Asia Conference on Information Systems, vol. 13, Langkawi, July 2017.

[7] C.-H. Liao, L.-X. Chen, J.-C. Yang, and S.-M. Yuan, "A photo post recommendation system based on topic model for improving facebook fan page engagement," Symmetry, vol. 12, no. 7, p. 1105, 2020.

[8] S. Carta, A. S. Podda, D. R. Recupero, R. Saia, and G. Usai, "Popularity prediction of instagram posts," Information, vol. 11, no. 9, p. 453, 2020. 
[9] M. B. Hajhmida and O. Oueslati, "Predicting mobile application breakout using sentiment analysis of facebook posts," Journal of Information Science, Article ID 0165551520917099, 2020.

[10] M. Lalmas, J. Lehmann, G. Shaked, F. Silvestri, and G. Tolomei, "Promoting positive post-click experience for instream yahoo gemini users," in Proceedings of the 21th ACM SIGKDD International Conference on Knowledge Discovery and Data Mining, pp. 1929-1938, Association for Computing Machinery, New York, NY, USA, August 2015.

[11] N. Silberstein, O. Somekh, Y. Koren et al., "Ad close mitigation for improved user experience in native advertisements," in Proceedings of the 13th International Conference on Web Search and Data Mining, pp. 546-554, Association for Computing Machinery, New York, NY, USA, January 2020.

[12] S. An, G. Kerr, and H. S. Jin, "Recognizing native ads as advertising: attitudinal and behavioral consequences," Journal of Consumer Affairs, vol. 53, no. 4, pp. 1421-1442, 2019.

[13] S. Youn and S. Kim, "Newsfeed native advertising on Facebook: young millennials' knowledge, pet peeves, reactance and ad avoidance," International Journal of Advertising, vol. 38, no. 5, pp. 651-683, 2019.

[14] O. Appiah, "Rich media, poor media: the impact of audio/ video vs. text/picture testimonial ads on browsers' evaluations of commercial web sites and online products," Journal of Current Issues \& Research in Advertising, vol. 28, no. 1, pp. 73-86, 2006.

[15] L. Spalding, S. Cole, and A. Fayer, "How rich-media video technology boosts branding goals," Journal of Advertising Research, vol. 49, no. 3, pp. 285-292, 2009.

[16] K. Goodrich, S. Schiller, and D. Galletta, "Intrusiveness of online video advertising and its effects on marketing outcomes," in Proceedings Of Thirty Second International Conference On Information Systems, Shangaiin-progress, Shangai, 2011.

[17] R. F. Otondo, J. R. Van Scotter, D. G. Allen, and P. Palvia, "The complexity of richness: media, message, and communication outcomes," Information \& Management, vol. 45, no. 1, pp. 21-30, 2008.

[18] X. Dong and P.-L. P. Rau, "Context awareness and perceived interactivity in multimedia computing," in Proceedings of 13th International Conference on Human-Computer Interaction, Berlin, Heidelberg, July 2009.

[19] N. Vallina-Rodriguez, J. Shah, A. Finamore et al., "Breaking for commercials: characterizing mobile advertising," in Proceedings of the 2012 Internet Measurement Conference, pp. 343-356, ACM, Boston, November 2012.

[20] K.-W. Su, P.-H. Huang, P.-H. Chen, and Y.-T. Li, "The impact of formats and interactive modes on the effectiveness of mobile advertisements," Journal of Ambient Intelligence and Humanized Computing, vol. 7, no. 6, pp. 817-827, 2016.

[21] I. L. Wu and W. H. Hsiao, "Involvement, content and interactivity drivers for consumer loyalty in mobile advertising: the mediating role of advertising value," International Journal of Mobile Communications, vol. 15, no. 6, pp. 577-603, 2017.

[22] S. S. Krishnan and R. K. Sitaraman, "Understanding the effectiveness of video ads: a measurement study," in Proceedings of the 2013 Conference on Internet Measurement Conference, pp. 149-162, ACM, Barcelona, Spain, October 2013.

[23] M. Pashkevich, S. Dorai-Raj, M. Kellar, and D. Zigmond, "Empowering online advertisements by empowering viewers with the right to choose," Journal of Advertising Research, vol. 52, no. 4, pp. 451-457, 2012.
[24] J. Lee and H. Lee, "Canonical correlation analysis of online video advertising viewing motivations and access characteristics," New Media \& Society, vol. 14, no. 8, pp. 1358-1374, 2012.

[25] B. Eyada and A. Milla, "Native advertising: challenges and perspectives," Journal of Design Sciences and Applied Arts, vol. 1, no. 1, pp. 67-77, 2020.

[26] N. Barbieri, F. Silvestri, and M. Lalmas, "Improving post-click user engagement on native ads via survival analysis," in Proceedings of the 25th International Conference on World Wide Web, pp. 761-770, Montreal, Canada, January 2017.

[27] A. Aribarg and E. M. Schwartz, "Native advertising in online news: tradeoffs among clicks, brand recognition and website trustworthiness," Brand Recognition and Website Trustworthiness, vol. 57, no. 1, pp. 20-34, 2019.

[28] B. W. Wojdynski and N. J. Evans, "Going native: effects of disclosure position and language on the recognition and evaluation of online native advertising," Journal of Advertising, vol. 45, no. 2, pp. 157-168, 2016.

[29] C. Campbell and N. J. Evans, "The role of a companion banner and sponsorship transparency in recognizing and evaluating article-style native advertising," Journal of Interactive Marketing, vol. 43, pp. 17-32, 2018.

[30] M. Lin, K. A. I. Tang, and Q. Fan, "Research on the impact of video-based native ads on users' purchase intention under the perceived value theory," in 6th International Conference on Humanities and Social Science Research (ICHSSR 2020), pp. 307-311, Atlantis Press, Hangzhou, China, April 2020.

[31] D. G. Goldstein, R. P. McAfee, and S. Suri, "The effects of exposure time on memory of display advertisements," in Proceedings of the 12th ACM Conference on Electronic Commerce-EC'11, p. 49, June 2011.

[32] R. G. Peters and T. H. Bijmolt, "Consumer memory for television advertising: a field study of duration, serial position, and competition effects," Journal of Consumer Research, vol. 23, pp. 362-372, 1997.

[33] H. Li and H.-Y. Lo, "Do you recognize its brand? The effectiveness of online in-stream video advertisements," Journal of Advertising, vol. 44, no. 3, pp. 208-218, 2015.

[34] C.-H. Cho and H. J. Cheon, "Why do people avoid advertising on the internet?" Journal of Advertising, vol. 33, no. 4, pp. 89-97, 2004.

[35] C. Bebk, L. M. Sciulli, and P. Bhagat, "Using eye tracking to assess the impact of advertising appeals on donor behavior," Journal of Nonprofit \& Public Sector Marketing, vol. 26, no. 4, pp. 354-371, 2014.

[36] M. Katz, B. Campbell, and Y. Liu, "Local and organic preference: logo versus text," Journal of Agricultural and Applied Economics, vol. 51, no. 02, pp. 328-347, 2019.

[37] Q. Li, Z. Huang, K. Christianson, and K. Christianson, "Visual attention toward tourism photographs with text: an eye-tracking study,” Tourism Management, vol. 54, pp. 243-258, 2016.

[38] R. Pieters and M. Wedel, "Attention capture and transfer in advertising: brand, pictorial, and text-size effects," Journal of Marketing, vol. 68, no. 2, pp. 36-50, 2004.

[39] M. Viswanathan, C. J. Torelli, L. Xia, and R. Gau, "Understanding the influence of literacy on consumer memory: the role of pictorial elements," Journal of Consumer Psychology, vol. 19, no. 3, pp. 389-402, 2009.

[40] K. A. Provins and P. Cunliffe, "The relationship between E.E.G. Activity and handedness," Cortex, vol. 8, no. 2, pp. 136-146, 1972.

[41] V. Venkatraman, A. Dimoka, P. A. Pavlou et al., "Predicting advertising success beyond traditional measures: new insights 
from neurophysiological methods and market response modeling," Journal of Marketing Research, vol. 52, no. 4, pp. 436-452, 2015.

[42] M. M. Bradley and P. J. Lang, "Measuring emotion: the selfassessment manikin and the semantic differential," Journal of Behavior Therapy and Experimental Psychiatry, vol. 25, no. 1, pp. 49-59, 1994.

[43] E. Du Plessis, "Recognition versus recall," Journal of Advertising Research, vol. 34, pp. 75-92, 1994.

[44] A. Poole and L. J. Ball, "Eye tracking in HCI and usability research," in Proceedings of Encyclopedia of Human Computer Interaction, pp. 211-219, IGI Global, Ghaoui. PV, USA, January 2006.

[45] J. A. Coan and J. J. B. Allen, "Frontal EEG asymmetry as a moderator and mediator of emotion," Biological Psychology, vol. 67 , no. 1-2, pp. 7-50, 2004.

[46] H. Blaiech, M. Neji, A. Wali, and A. M. Alimi, "Emotion recognition by analysis of EEG signals," in Proceedings of 13th International Conference on Hybrid Intelligent Systems (HIS 2013), pp. 312-318, IEEE, Gammarth, Tunisia, December 2013.

[47] W.-H. Hsiao and T.-S. Chang, "Understanding consumers' continuance intention towards mobile advertising: a theoretical framework and empirical study," Behaviour \& Information Technology, vol. 33, no. 7, pp. 730-742, 2013.

[48] R. L. Hodes, E. W. Cook, and P. J. Lang, "Individual differences in autonomic response: conditioned association or conditioned fear?" Psychophysiology, vol. 22, no. 5, pp. 545560, 1985. 